\title{
Infecciones bacterianas del tracto reproductivo masculino y su papel en la fertilidad
}

\author{
Jenniffer Puerta-Suárez ${ }^{1}$, Mariluz Giraldo ${ }^{2}$, Ángela Patricia Cadavid ${ }^{1}$, Walter Cardona- \\ Maya ${ }^{1}$. \\ ${ }^{1}$ Grupo Reproducción, Facultad de Medicina, Universidad de Antioquia, Medellín, Colombia. ${ }^{2}$ IPS Universitaria, \\ Universidad de Antioquia, Medellín, Colombia.
}

\section{RESUMEN}

Antecedentes: El espermatozoide es una célula altamente especializada encargada de llevar el material genético paterno hasta el tracto reproductivo femenino en búsqueda del oocito, no obstante durante su desplazamiento puede interactuar con sustancias, otras células e incluso microorganismos que puede transportar desencadenando procesos infecciosos que alteran el éxito reproductivo. Objetivo: Describir las bacterias involucradas en la alteración de la función reproductiva y sus efectos sobre calidad espermática, así como la capacidad de los espermatozoides para transportar infecciones y diseminarlas al tracto reproductivo femenino. Resultados: Las infecciones bacterianas que afectan el tracto reproductivo masculino se clasifican en infecciones de transmisión sexual, del tracto urinario y las asociadas a la microbiota bacteriana, donde están implicadas una gran variedad de agentes etiológicos como Chlamydia trachomatis, Ureoplasma urealyticum, Mycoplasma hominis, Escherichia coli y los Staphyloccoccus coagulasa negativos. Aún es controversial el efecto de estos gérmenes sobre los parámetros seminales así como la presencia de microbiota en el semen; su diagnóstico depende de la calidad de la muestra, de la sensibilidad de la técnica de detección y de los factores de riesgo que presente el individuo. Conclusión: Los procesos inflamatorios e infecciosos en el trato reproductivo masculino influyen en la fertilidad, por lo que se requiere profundizar en el estudio de estos procesos, establecer más y mejores métodos diagnósticos y pautas para el autocuidado que disminuyan la propagación de estos agentes patógenos.

\section{PALABRAS CLAVE: Infección, inflamación, infertilidad, espermatozoides, microorganismos, bacterias}

\section{SUMMARY}

Introduction: Spermatozoon is a highly specialized cell responsible for carrying the paternal genetic material toward the oocyte. During its journey in the female reproductive tract, the sperm cell interacts with substances, cells and also microorganisms that can be transported, thus triggering infectious processes that could alter reproductive success. Objective: Describe the species of bacteria involved in the alteration of sperm cells reproductive function and their effect on the sperm quality. In addition, evaluate the ability of the sperm cell to carry infections and disseminate them in the female reproductive tract. Results: Bacterial infections that affect the male reproductive tract are classified as sexually transmitted infections, urinary tract infections, and infections associated with bacterial microbiome. Chlamydia trachomatis, Ureoplasma urealyticum, Mycoplasma hominis, Escherichia coli and coagulase-negative Staphylococcus are the main etiologic 
agents of these infections. The presence of bacteria in semen and their effect of in semen parameters are still controversial. To probe that bacteria are present in semen depends on the quality of the sample, the sensitivity and specificity of the detection technique and the patient risk factors. Conclusion: Inflammatory and infectious processes in the male reproductive treatment affect fertility; therefore, further study of the role of thoseprocesses, establishment of more and better diagnostic methods and guidelines for self-care to decrease the spread of these pathogens will be necessary.

\section{KEY WORDS: Infection, inflammation, infertility, sperm, microorganisms, bacteria}

\section{INTRODUCCIÓN}

El cuerpo humano es una aglomeración de células que se especializan para cumplir diversas funciones partiendo de dos gametos fundamentales para el desarrollo de la vida: el oocito y el espermatozoide. Durante el desarrollo de un nuevo individuo, estas células especializadas que comparten un origen común, desde el momento en que el espermatozoide transfiere la información genética paterna a su homólogo femenino, logran agruparse para formar tejidos que integrarán órganos con una comunicación estrecha la cual posibilita su funcionamiento armonioso.

De otro lado, los microorganismos participan durante el desarrollo y el proceso adaptativo al ambiente, los cuales alcanzan a cubrir cerca del $90 \%$ del cuerpo humano (1), cumpliendo diversas funciones como lo relacionado con la absorción de nutrientes y la protección por competición contra la invasión por patógenos. Sin embargo, el carácter entrópico de la vida y la disponibilidad de nutrientes, hacen que exista cierto tropismo de los microorganismos por algunos sitios anatómicos y que algunos virus, bacterias, hongos e incluso parásitos sean responsables de procesos infecciosos en el organismo (2).

Dentro de los sitios anatómicos colonizados por microorganismos se encuentra el tracto reproductivo, donde se desarrollan las infecciones de transmisión sexual (ITS) las cuales han sido tradicionalmente, de mayor relevancia en mujeres que hombres. Los mecanismos de control y las estrategias de prevención, promoción y restablecimiento de las condiciones de salud sexual y reproductiva se han orientado a la población femenina con ejemplos tan claros como la implementación de la citología para el diagnóstico de neoplasias asociadas a la infección por el virus del papiloma humano (VPH), tomando medidas de prevención como la aplicación de la vacuna contra este agente etiológico principalmente en mujeres adolescentes. En contraste pocas son las estrategias de acción en hombres, lo que finalmente ha relegado no solo su estado de salud, sino su papel al actuar como reservorio de un alto número de microorganismos de forma crónica e incluso subclínica (3).

Con el objetivo de rescatar el papel que cumple el hombre en la transmisión de microorganismos para su inclusión en programas de salud y resaltar el papel de la célula espermática como posible vehículo de patógenos, esta revisión se enfocará de forma especial en los microorganismos involucrados en la afección de la función reproductiva, la calidad espermática y los problemas de fertilidad asociados, así como en la capacidad de la célula espermática para transportar infecciones y diseminarlas al tracto reproductivo femenino.

\section{INFECCIONES BACTERIANAS DEL TRACTO REPRODUCTIVO MASCULINO}

Las infecciones del tracto reproductivo masculino se pueden clasificar en tres tipos: i) las infecciones de transmisión sexual, ii) las infecciones del tracto urinario, debido a que ambos tractos, genital y urinario, comparten varias porciones anatómicas, y iii) las infecciones asociadas a desequilibrios en la microbiota bacteriana.

Un gran número de bacterias puede colonizar el tracto reproductivo masculino y desencadenar procesos infecciosos con múltiples consecuencias. Este tipo de infecciones se caracterizan por ser comunes pero generalmente autolimitadas y en la gran mayoría de los casos asintomáticos, lo que le da el carácter de infección crónica acompañada en algunas ocasiones de inflamación persistente, que puede finalmente generar alteraciones en la fertilidad (2). Estas alteraciones pueden desarrollarse bien sea por daño directo de la célula espermática o de su nicho, o como vehículo para el ascenso de un gran número de microorganismos en el tracto reproductivo femenino lo que promovería procesos infecciosos e inflamatorios como es el caso de la enfermedad inflamatoria pélvica. 


\section{Infecciones de transmisión sexual}

El término de ITS hace referencia a la infección transmitida de persona a persona por ciertos microorganismos que con alta frecuencia emplean el contacto sexual durante las relaciones sexo-genitales donde se incluye el sexo vaginal, el anal y el oral (4). Cada año se reportan alrededor de 448 millones de nuevos casos de ITS en el mundo (4), entre los que se destacan las virales como el virus de la inmunodeficiencia humana (VIH), VPH y el virus de la hepatitis $B$, además de bacterianas como las responsables de la gonorrea, la clamidiasis y la sífilis. A pesar que la gran mayoría de ITS son prevenibles y curables, las medidas implicadas en su resolución aumentan los costos en salud, con implicaciones sociales, económicas y personales (5). Entre las bacterias involucradas en las infecciones del tracto genital masculino más comunes se encuentran Chlamydia trachomatis, Neisseria gonorrhoeae, Treponema pallidum, Ureaplasma urealyticum y Mycoplasma hominis (6), sin embargo las dos últimas no son ampliamente consideradas como ITS debido a su presencia en individuos sanos y asintomáticos.

Chlamydia trachomatis. Es una célula gram negativa, intracelular obligada cuyo único reservorio es el humano y es catalogada como el principal agente causal de uretritis, cervicitis mucopurulenta, enfermedad pélvica inflamatoria, infertilidad tubal y epididimitis (7). Es la bacteria causante de ITS más prevalente en el mundo y puede causar enfermedad ocular grave durante el paso del niño a través del canal vaginal durante el parto (8). Presenta un ciclo de vida en el cual interactúan dos morfologías: i) el cuerpo elemental el cual es la forma extracelular e infecciosa que se transmite a través de secreciones que entran en contacto con la mucosa lesionada, $y$ ii) el cuerpo reticular o forma replicativa intracelular que se multiplica y diferencia nuevamente a cuerpo elemental para infectar nuevas células (6). Esta infección bacteriana reporta una incidencia de aproximadamente 100 millones de nuevos casos diagnosticados cada año (9), y en Colombia tiene una prevalencia del $5 \%$ en mujeres bogotanas (10) y del $5,9 \%$ en mujeres de la ciudad de Montería (11), con alto subregistro por la ausencia de tamización, siendo un problema aún mayor en hombres en los que a pesar de presentar una prevalencia similar a la reportada en mujeres, no suelen implementarse estrategias de control (12). Este microorganismo se ha asociado con infertilidad masculina aunque con resultados controversiales y algunos estudios sugieren que la infección por $\mathrm{C}$. trachomatis reduce la movilidad, la viabilidad, la concentra- ción y la morfología espermática $(6,8)$.

Neisseria gonorrhoeae. Este diplococo gramnegativo, oxidasa positivo, móvil no esporulado, es la principal causa de uretritis en hombres, además de estar implicado en prostatitis y en epididimitis (13). Sólo se hospeda en el humano, para lo cual posee mecanismos que le permite la adherencia a las células epiteliales y su transmisión se da por contacto directo con mucosa del tracto genital (14). N. gonorrhoeae presenta una estructura denominada pili, la cual tiene la habilidad de generar hemaglutinación permitiendo la adherencia a los eritrocitos, además de mediar la interacción entre las células epiteliales, células de la mucosa uretral, leucocitos y espermatozoides en ensayos in vitro, donde se ha encontrado la bacteria unida a varias porciones de la célula espermática influenciada por una variedad de factores físicos y químicos (15), sin embargo, Liu y cols reportan que la infección por este microorganismo no altera ni la viabilidad ni la movilidad espermática (16).

Treponema pallidum. Sífilis es la denominación que se le da a la ITS causada por esta bacteria, la cual es de difícil diagnóstico por las diversas manifestaciones clínicas que presenta (17), esta espiroqueta mide entre 5 y $20 \mu \mathrm{m}$ de largo y es altamente contagiosa, se ha reportado su presencia en el espacio intracelular de los fibroblastos, de las células de Leydig, de células intersticiales y de los espermatocitos, mediante observaciones al microscopio electrónico en testículos de conejo (18), por lo que puede alterar el normal desarrollo de la espermatogénesis.

Mycoplasmas. Dentro de este género se encuentran principalmente Ureoplasma urealyticum y Mycoplasma hominis. Son microorganismos extracelulares que se adhieren a superficies epiteliales, de vida libre, con un genoma pequeño por la pérdida de algunas rutas metabólicas y que requieren medios complejos para su aislamiento (14), infectan con mayor frecuencia el aparato genital y se han implicado en el desarrollo de vaginosis bacteriana, septicemia posparto, uretritis y aborto (19). Se han considerado como microbiota genital, sin embargo y particularmente, U. urealyticum se encuentra en el $70 \%$ de las personas sexualmente activas por lo que se ha asociado al grupo de las ITS. El hallazgo de estas bacterias en el eyaculado afecta la concentración y la morfología espermática (6), se cree que tiene la capacidad de interactuar con los espermatozoides y desencadenar alteraciones en la fertilidad masculina por lo que se recomienda realizar la búsqueda de estos microorganismos de forma rutinaria durante el análisis seminal (20). DiazGarcía y cols, observaron luego de 10 minutos de 
infección, pequeñas colonias de Mycoplasma hominis unidas a los espermatozoides en la cabeza, pieza intermedia y cola, sin embargo no se observaron patrones específicos de interacción, además las dosis mínimas efectivas encontradas difieren de acuerdo al tipo de muestra, concluyendo que esta bacteria tiene la capacidad de parasitar la cabeza y la pieza intermedia de los espermatozoides (19).

\section{Infecciones del tracto urinario}

Las infecciones del tracto urinario son clasificadas de acuerdo a la porción anatómica que afectan ya sea en tracto urinario bajo o alto, además se ha considerado que cada agente etiológico presenta determinado tropismo por sitios anatómicos, de acuerdo a la alta frecuencia con la que se diagnostican en las diferentes situaciones clínicas, como puede observarse en la Tabla I. Los síntomas de infección urinaria incluyen micción dolorosa y frecuente, además en los casos de infecciones urinarias del tracto alto los síntomas también pueden incluir fiebre y dolor localizado (21).

Una infección poco usual del tracto urinario es la tuberculosis de localización extra pulmonar causada por Mycobacterium tuberculosis la cual puede afectar tanto la vejiga y la uretra, como el epidídimo, el testículo, los conductos deferentes, la vesícula seminal, la próstata y los conductos eyaculatorios (26). Esta infección puede alterar la espermatogénesis, donde la manifestación clínica más común en pacientes asintomáticos es la infertilidad. El daño generado por este microorganismo se genera por la obstrucción anatómica ocasionada por la formación de los granulomas y la fibrosis de los tejidos del tracto reproductivo además de la inflamación generada, por lo que la mayoría de los pacientes con infertilidad por tuberculosis requieren técnicas de reproducción asistida para alcanzar el éxito reproductivo (27). La manifestación clínica más importante durante la tuberculosis del tracto urinario es la azoospermia. Sole-Balcells y cols, en un estudio en 50 pacientes con diagnóstico de tuberculosis urinaria demostraron que todos los que presentaban alteraciones en las estructuras genitales mostraron modificaciones en la morfología espermática y el $75 \%$ de los pacientes sin lesiones genitales presentaron oligoastenozoospermia, la cual no presenta mejoría post tratamiento (28).

\section{Infecciones asociadas a la microbiota bacteriana}

Algunos sitios anatómicos presentan un recubrimiento especial con bacterias conocidos como microorganismos saprófitos, los cuales se aprovechan de los nutrientes que les proporciona el hospedero sin causarle daño alguno; estos microorganismos actúan por inhibición competitiva y ocupación de un sitio anatómico determinado en los cuales generalmente impiden la colonización por otros microorganismos patógenos que puedan alterar la arquitectura e integridad de los epitelios, sin embargo cuando hay alteraciones en el organismo, como las generadas por los cambios hormonales durante el ciclo menstrual femenino o la alteración en la cantidad de microorganismos ocupando un sitio anatómico por la escasez de nutrientes, la relación microorganismo - humano puede tornarse de mutualista a parasitaria, desencadenando en algunas ocasiones procesos infecciosos, como es el caso de la vaginosis bacteriana causada por Gardnerella vaginalis debido al desequilibrio en la microbiota de la vagina por una disminución de Lactobacillus spp (29). Un ejemplo de esta relación la establecen los cocos grampositivos, los cuales en su mayoría hacen parte de la microbiota normal de la piel y de gran cantidad de sitios anatómicos, entre ellos especialmente los denominados Staphyloccoccus coagulasa negativos como $\mathrm{S}$. saprophyticus y $\mathrm{S}$. epidermidis, relacionados ampliamente con infecciones del tracto urinario, pero solo se establecen como importantes durante el diagnóstico cuando el paciente presenta una sintomatología claramente definida (30) o un recuento mayor a $10^{5} \mathrm{UFC} / \mathrm{mL}$ en cultivos cuantitativos de orina. Otro coco grampositivo presente en este tipo de infecciones es Staphylococcus aureus el cual se ha relacionado con el aumento en el número de espermatozoides que sufren apoptosis (31).

Aunque son muchos los ejemplos que pueden citarse con respecto a infecciones relacionadas con alteraciones en la microbiota genital, un microorganismo que cobra gran importancia en la embarazadas es el Streptococcus agalactiae, relacionado con la ruptura prematura de membranas, razón por la que se realizan tamizaciones de este microorganismo como parte de los controles prenatales. Adicionalmente, Vieira y cols, resaltan la importancia de desarrollar anticonceptivos con efecto deletéreo sobre este tipo de microorganismos para impedir la colonización femenina que pueda afectar el desarrollo de un futuro embarazo (32). 
Tabla I

ETIOLOGÍA DE LAS INFECCIONES DEL TRACTO URINARIO

\begin{tabular}{|c|c|c|c|}
\hline \multicolumn{2}{|c|}{ Sitio anatómico } & Situación clínica & Agente etiológico \\
\hline \multirow{4}{*}{ 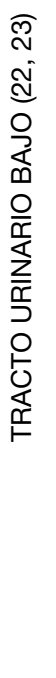 } & \multirow[t]{2}{*}{ Uretra } & Uretritis & $\begin{array}{l}\text { Escherichia coli } \\
\text { Chlamydia } \\
\text { Neisseria gonorrhoeae }\end{array}$ \\
\hline & & Síndrome uretral & $\begin{array}{l}\text { Candida spp } \\
\text { Trichomonas } \\
\text { Virus del herpes simple }\end{array}$ \\
\hline & Vejiga & Cistitis & $\begin{array}{l}\text { Escherichia coli } \\
\text { Klebsiella y Proteus } \\
\text { Staphylococcus saprophyticus* } \\
\text { Serratia marcescens } \\
\text { Pseudomonas aeruginosa } \\
\text { Enterococcus faecalis }\end{array}$ \\
\hline & Próstata & Prostatitis & $\begin{array}{l}\text { Enterococcus spp. } \\
\text { Escherichia coli } \\
\text { Klebsiella pneumoniae } \\
\text { Proteus mirabilis } \\
\text { Pseudomona aeruginosa } \\
\text { Staphylococcus aureus }\end{array}$ \\
\hline \multirow{4}{*}{ 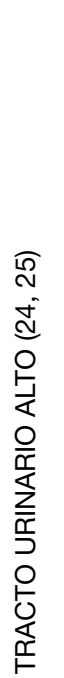 } & Uréteres & Ureteritis & $\begin{array}{l}\text { Neisseria gonorrhoeae } \\
\text { Chlamydia trachomatis } \\
\text { Mycoplasma genitalium }\end{array}$ \\
\hline & \multirow{3}{*}{ Riñón } & Pielitis y pielonefritis enfisematosa & $\begin{array}{l}\text { Corynebacterium urealyticum } \\
\text { Escherichia coli } \\
\text { Klebsiella spp. } \\
\text { Proteus spp. }\end{array}$ \\
\hline & & Pielonefritis aguda & $\begin{array}{l}\text { Escherichia coli } \\
\text { Klebsiella spp. } \\
\text { Proteus spp. } \\
\text { Enterococcus spp. } \\
\text { Streptococcus agalactiae } \\
\text { Staphylococcus spp. }\end{array}$ \\
\hline & & Glomerulonefritis & $\begin{array}{l}\text { Streptococcus pyogenes } \\
\text { Staphylococcus spp. } \\
\text { Salmonella spp. } \\
\text { Mycoplasma spp. } \\
\text { Klebsiella spp. }\end{array}$ \\
\hline
\end{tabular}

${ }^{*}$ Asociado a catéter.

\section{MOLÉCULAS IMPLICADAS EN LA INTERACCIÓN ENTRE ESPERMATOZOIDESY BACTERIAS}

Durante el desarrollo de todo proceso infeccioso, los puentes de comunicación entre los microorganismos y sus células hospederas son esenciales, debido a que determinarán el éxito del proceso de colonización o de contingencia de la infección.
Los microorganismos presentan una serie de moléculas que le permiten la supervivencia en diversas partes anatómicas donde las secreciones podrían eliminarlos, como es el caso del pili bacteriano, estructura delgada que le permite a las bacterias que lo poseen adherirse a otras células.

Las células del hospedero humano expresan moléculas con amplia diversidad de funciones para 
la defensa contra patógenos, una de ellas es alertar al sistema inmune cuando se compromete el equilibrio del organismo. Un ejemplo clásico de este tipo de moléculas son receptores de reconocimiento de patógenos o tipo toll (TRL), los cuales están presentes en las células de Sertoli y tiene la capacidad de producir mediadores de inflamación, que protegen al organismo de una infección microbiana ascendente (33), pero durante esta acción de defensa también se pueden generar especies reactivas del oxígeno (ERO) las cuales limitan la función de la célula espermática. El mecanismo de acción de estas sustancias, que se desencadenan por la presencia de bacterias y leucocitos, se basa en la generación de un desbalance oxidativo en donde la acumulación de leucocitos está asociada con fagocitosis que luego causa la activación de receptores y vías de transducción generando la activación biológica de citoquinas proinflamatorias que modulan negativamente el sistema antioxidante $(21,32)$.

El papel de las citoquinas proinflamatorias puede ser altamente perjudicial para las células espermáticas, por ejemplo la interleuquina 1 (IL1) está presente en altas concentraciones en el plasma seminal de pacientes infértiles, aunque su presencia no se ha relacionado con afectación de la movilidad espermática ni del proceso de reacción acrosomal La IL6 se ha asociado con lipoperoxidación y producción de ERO con mayor frecuencia en hombres infértiles.Las altas concentraciones de IL8 se han asociado con alteración de la movilidad espermática y con inhibición de la ATPasa de sodio y potasio, la superóxido dismutasa y el aumento en la producción de óxido nítrico. Finalmente el TNFa incrementa la externalización de la fosfatidilserina y la fragmentación del DNA con lo que se afecta directamente la calidad espermática y por ende se disminuye la probabilidad de éxito reproductivo (21).

\section{METODOLOGÍAS EMPLEADAS PARA EL DIAG- NÓSTICO DE INFECCIONES DEL TRACTO RE- PRODUCTIVO MASCULINO}

El éxito en el diagnóstico microbiológico proviene en gran medida de la calidad de la muestra que se obtenga para el análisis, sin embargo, la investigación microbiológica del tracto urogenital masculino se torna compleja debido a los múltiples componentes del eyaculado y a los múltiples microorganismos saprófitos que pueden encontrarse. La identificación microbiológica debe enfocarse en la búsqueda de bacterias gram negativas como Escherichia coli, Klebsiella sp. y Proteus sp.; bacterias gram positivas como Enterococcus sp, Staphylococcus aureus; bacterias anaerobias y otros patógenos aun controversiales como Staphylococcus haemolyticus, Staphylococcus epidermidis, Mycoplasma hominis (21).

Como en todas las pruebas microbiológicas, se debe tener cuidado especial con la generación de falsos positivos o negativos, por ejemplo por la presencia de gérmenes en sitios genitales expuestos como el glande, el prepucio y con las manos del paciente cuando éste obtiene la muestra de semen, o por el contrario, por sustancias inhibitorias de microorganismos. Debido a que el semen es una mezcla de fluidos de varias glándulas y de células espermáticas, el cultivo detecta la presencia de bacterias pero no discrimina el sitio de infección. Algunos autores recomiendan el uso de diluciones del fluido seminal en solución salina antes de proceder a la siembra en agar para mejorar la detección de microorganismos como Neisseria gonorrhoeae $(21,34)$. Durante la interpretación de los resultados es conveniente evaluar los factores de riesgo del individuo y su susceptibilidad a sufrir infecciones, entre los que se encuentran el estilo de vida, la promiscuidad, la higiene, los hábitos intestinales, factores médicos o clínicos que se asocien con infecciones complicadas del tracto urinario, infecciones de las glándulas accesorias y parámetros espermáticos convencionales y no convencionales. Aunque no hay criterios diagnósticos totalmente eficaces para este tipo de infecciones, un cultivo cuantitativo del semen o de orina con recuentos superiores a $10^{3}$ unidades formadoras de colonias (UFC) para microorganismos catalogados como patógenos o mayores a $10^{5}$ para microorganismos saprofitos en presencia de sintomatología, puede acercarse al diagnóstico (35). Finalmente, una adecuada evaluación de los procesos inflamatorios e infecciosos de esta región anatómica debe considerar la composición bioquímica del semen o de la orina, el componente microbiológico y la presencia de leucocitos (21). El frotis uretral parece ser apropiado para el diagnóstico de procesos infecciosos teniendo en cuenta que el paciente debe tener una abstinencia sexual de por lo menos 24 horas y de micción de por lo menos 3 horas y en todos los casos debe suspender el uso de medicamentos, especialmente antibióticos con una semana de anterioridad (21). 


\section{RELACIÓN ENTRE LA INFERTILIDAD Y LAS IN- FECCIONES DEL TRACTO GENITOURINARIO MASCULINO}

La infertilidad masculina es una entidad médica frecuente que compromete aproximadamente a uno de cada 20 hombres y en la que las infecciones del tracto reproductivo son consideradas como la principal causa etiológica (33). Entre el 5 y el $12 \%$ de pacientes atendidos en las clínicas de infertilidad que cursan con procesos inflamatorios relacionados con infecciones, se ha reportado daño en la calidad espermática con reducción de la movilidad, la concentración y la morfología espermática, e incluso se ha encontrado la presencia de anticuerpos antiespermatozoides en el $8 \%$ de esta población (20).

Adicionalmente, se ha reportado la presencia de estos anticuerpos en sangre, semen o inclusive en secreciones cervicovaginales en parejas infértiles cuya causa de infertilidad parece ser inmunológica, asociada a trastornos en mecanismos inmunomoduladores que alteran la funcionalidad del espermatozoide e inhiben su capacidad de interacción con el oocito (20).

El desarrollo de la infertilidad masculina originada por procesos infecciosos se ha relacionado con múltiples causas, entre ellas la inflamación crónica puede afectar la arquitectura y morfología testicular (28) y del epidídimo, lo que altera la correcta formación y capacitación del espermatozoide para cumplir su objetivo; es por eso que para el desarrollo de la espermatogénesis el testículo debe ser un órgano inmunológicamente privilegiado, para que los procesos infecciosos o inflamatorios no alteren las células germinales.

El espermatozoide tiene la capacidad de interactuar con células epiteliales y microorganismos lo que puede afectar la calidad espermática en parámetros como el volumen, la concentración y la viabilidad, e incluso sobre otras características no evaluadas de forma rutinaria como la formación de ERO, la integridad de la cromatina y el desencadenamiento de procesos apoptóticos $(36,37)$, que finalmente pueden afectar el proceso reproductivo; además en infecciones prostáticas se ha encontrado disminución del volumen seminal, de la fructosa y de la alfa glucosilasa, segregados durante la eyaculación (20).

Las técnicas de reproducción asistida son ampliamente usadas en la actualidad, por ejemplo, algunos pacientes con cáncer almacenan espermatozoides antes de la quimioterapia para cumplir con sus deseos de concepción, por lo que en el campo de la infertilidad masculina el cultivo de muestras de semen tiene dos aplicaciones, la primera de ellas relacionada con la búsqueda del agente causal de infertilidad cuando esta se debe a procesos infecciosos, la segunda, la evaluación de aquellas muestras que van a ser empleadas en técnicas de reproducción asistida (38). Para la criopreservación del semen y su posterior uso en técnicas de reproducción asistida, se recomienda un tamizaje del estado serológico frente a microorganismos como el $\mathrm{VIH}$ y el virus de la hepatitis $\mathrm{B}$ y $\mathrm{C}$, debido a que se ha demostrado que algunos virus pueden interactuar con la célula espermática, aunque en la mayoría de los casos no se han descrito los receptores implicados (39) y contra Treponema pallidum, sin embargo, aún no se tienen en consideración otros microorganismos que puede alojar este fluido biológico (38).

Las muestras de semen empleadas para técnicas de reproducción asistida deben ser liberadas de los microorganismos presentes, bien sean considerados como patógenos o saprofitos, que como se describió anteriormente, afectan la calidad espermática. Una opción para eliminar estos microorganismos es el empleo de antibióticos, sin embargo, altas concentraciones de estas drogas muestran un efecto dañino sobre los espermatozoides e incluso pueden afectar la implantación del embrión (38).

\section{CONCLUSIONES}

La relación entre la infección, la inflamación y la infertilidad está ampliamente demostrada y documentada por una gran variedad de autores $(8,35,40)$, aunque algunos mecanismos no están claramente dilucidados o incluso pueden ser divergentes entre los diferentes estudios. Estos procesos deben tomarse en consideración al momento del diagnóstico de infertilidad y de la elección de técnicas de reproducción asistida cuando éstas sean necesarias (27). Para el diagnóstico de procesos infecciosos en el tracto reproductivo masculino existen una gran variedad de técnicas, sin embargo, el uso de una sola puede no ser efectiva, por lo que una integración de pruebas parece ser más útil; además, se debe realizar una correcta interpretación de los factores de riesgo o protectores que presente el individuo frente a este tipo de procesos y como medida inicial asegurarse de la calidad de la muestra empleada en el diagnóstico para generar un adecuado análisis e interpretación de cada proceso infeccioso (21). De otro lado, en los casos de infertilidad idiopática en donde no se ha podido establecer un proceso infeccioso como agente causal, éste no debe descartarse debido a los inconvenientes que presentan actualmente las técnicas de 
diagnóstico de este tipo de problemas en el tracto reproductivo (35). Sólo un pequeño porcentaje de microorganismos puede ser identificado y cultivado en la actualidad, lo que establece un límite en nuestro conocimiento tanto de la microbiota como de microorganismos patógenos no solo del tracto reproductivo, sino del organismo y el medio ambiente en general. Establecer el contenido de microbiota en el semen puede contribuir a un mejor entendimiento de las relaciones establecidas entre estos microorganismos y la célula espermática, puesto que no puede descartarse que algunos de estos microorganismos puedan establecer relaciones benéficas con el espermatozoide, además, de tratar de entender como esta microbiota puede verse afectada por diferentes factores, lo que podría ayudar a establecer pautas para el autocuidado masculino frente a infecciones que van a impactar directamente sobre el estado de salud sexual y reproductivo femenino, comprendiendo y dando relevancia al papel que cumple el hombre en las infecciones de transmisión sexual. Finalmente, se debe resaltar la importancia de estudiar con mayor profundidad los procesos infecciosos e inflamatorios que se dan a nivel del tracto masculino, esto nos ayudaría a comprender aún más los factores implicados en el éxito reproductivo (38), a establecer más y mejores métodos diagnósticos, a disminuir los gastos económicos requeridos para restablecer el estado de salud sexual y reproductiva, a disminuir la transmisión de agentes patógenos que pueden ser transportados del tracto reproductivo masculino al femenino, e incluso al producto de la gestación y a establecer pautas para el autocuidado que disminuyan la propagación de estos agentes infecciosos, lo que se convierte en una estrategia de mayor impacto que sólo emplear recursos en el restablecimiento de la salud después de problemas infecciosos.

AGRADECIMIENTOS. A la Estrategia de Sostenibilidad 2013/2014 de la Universidad de Antioquia y a Colciencias (proyecto 111556933373 ) por el apoyo financiero. JPS es joven investigadora de Colciencias.

\section{REFERENCIAS}

1. Mandar R. Microbiota of male genital tract: impact on the health of man and his partner. Pharmacol Res 2013;69(1):32-41.

2. Dohle GR. Inflammatory-associated obstructions of the male reproductive tract. Andrologia 2003;35(5):321-4.

3. Cardona-Arias J, Puerta-Suárez J, Flórez-Duque J. Prevalencia del virus papiloma humano y sus factores de riesgo en hombres: revisión sistemática. Infectio
2011;15(4):268-76.

4. OMS. Infecciones de transmisión sexual. Nota descriptiva $\mathrm{N}^{\circ} 110$ de agosto de 2011. 2011; Disponible en: http://www.who.int/mediacentre/factsheets/fs110/ es/.

5. Lea T, Mao L, Bath N, Prestage G, Zablotska I, de Wit $\mathrm{J}$, et al. Injecting drug use among gay and bisexual men in Sydney: prevalence and associations with sexual risk practices and HIV and hepatitis C infection. AIDS Behav 2013;17(4):1344-51.

6. Rybar R, Prinosilova P, Kopecka V, Hlavicova J, Veznik Z, Zajicova A, et al. The effect of bacterial contamination of semen on sperm chromatin integrity and standard semen parameters in men from infertile couples.Andrologia 2012;44Suppl 1:410-8.

7. Hirano $\mathrm{Y}$, Shibahara $\mathrm{H}$, Koriyama J, Tokunaga $\mathrm{M}$, Shimada K, Suzuki M. Incidence of sperm-immobilizing antibodies in infertile women with past Chlamydia trachomatis infection. Am J Reprod Immunol 2011;65(2):127-32.

8. Cunningham KA, Beagley KW. Male genital tract chlamydial infection: implications for pathology and infertility. Biol Reprod 2008;79(2):180-9.

9. Mackern-Oberti JP, Motrich RD, Breser ML, Sanchez LR, Cuffini C, Rivero VE. Chlamydia trachomatis infection of the male genital tract: An update. J Reprod Immunol 2013; 100(1):37-53.

10. Molano M, Weiderpass E, Posso H, Morre SA, Ronderos $\mathrm{M}$, Franceschi $\mathrm{S}$, et al. Prevalence and determinants of Chlamydia trachomatis infections in women from Bogota, Colombia. Sex Transminfect 2003;79(6):474-8.

11. Alvis N, Mattar S, García J, Conde E, Díaz A. Infecciones de transmisión sexual en un grupo de alto riesgo de la ciudad de Montería, Colombia. Rev Salud Pública 2007;9(1):86-96.

12. Wagenlehner FM, Weidner W, Naber KG. Chlamydial infections in urology. World JUrology 2006;24(1):4-12.

13. Templeton DJ, Manokaran N, O'Connor CC. Prevalence and predictors of chlamydia co-infection among patients infected with gonorrhoea at a sexual health clinic in Sydney. Sex Health 2012;9(4):392-4.

14. Holmes K, Stamm W, Piot P, Wasserheit J, Corey L, Cohen M. Sexually Transmitted Diseases 4th ed. New York: McGraw; 2008.

15. Gomez Cl, Stenback WA, James AN, Criswell BS, Williams RP. Attachment of Neisseria gonorrhoeae to human sperm. Microscopical study of trypsin and iron. Br J Vener Dis1979;55(4):245-55.

16. Liu JH, Li HY, Cao ZG, Duan YF, Li Y, Ye ZQ. Influence of several uropathogenic microorganisms on human sperm motility parameters in vitro. Asian $\mathrm{J}$ Androl 2002;4(3):179-82.

17. Sun R, Lai DH, Ren RX, Lian S, Zhang HP. Treponema pallidum-specific antibody expression for the diagnosis of different stages of syphilis. Chinese Med J 2013;126(2):206-10.

18. Sykes JA, Miller JN. Intracellular location of Treponema pallidum (Nichols strain) in the rabbit testis. Infection and immunity. Infect Immun 1971;4(3):307-14. 
19. Diaz-Garcia FJ, Herrera-Mendoza AP, Giono-Cerezo $S$, Guerra-Infante FM. Mycoplasma hominis attaches to and locates intracellularly in human spermatozoa. Hum Reprod 2006;21(6):1591-8.

20. Andrade-Rocha FT. Ureaplasma urealyticum and Mycoplasma hominis in men attending for routine semen analysis. Prevalence, incidence by age and clinical settings, influence on sperm characteristics, relationship with the leukocyte count and clinical value. Urol Int 2003;71(4):377-81.

21. La Vignera S, Condorelli RA, Vicari E, Salmeri M, Morgia G, Favilla V, et al. Microbiological investigation in male infertility: a pratical overview. J Med Microbiol 2014;63:1-4.

22. Echevarría-Zarate J, Aguilar ES, Osores-Plenge F. Infección del tracto urinario y manejo antibiótico. Acta Med Per 2006;23(1):26-31.

23. Schiefer $\mathrm{H}$. Microbiology of male urethra adnexitis: diagnostic procedures and criteria for aetiologic classification. Andrologia 1998;30(S1):7-13.

24. Forrest JW, Jr., John F, Mills LR, Buxton TB, Moore WL, Jr., Hudson JB, et al. Immune complex glomerulonephritis associated with Klebsiella pneumoniae infection. Clin Nephrol 1977;7(2):76-80.

25. Melero MJ, Sarquis SG, Biancolini C, Baredes N, Villa R. Pielonefritis enfisematosa aguda bilateral: Un desafío terapéutico. Medicina (Buenos Aires) 2007;67(3):282-4.

26. Wise GJ, Shteynshlyuger A. An update on lower urinary tract tuberculosis.Curr Urol Rep 2008;9(4):30513.

27. Kumar R. Reproductive tract tuberculosis and male infertility. Indian J Urol 2008;24(3):392-5.

28. Sole-Balcells F, Jimenez-Cruz F, de Cabezon JS, Rosello AS. Tuberculosis and infertility in men. Eur Urol 1977;3(3):129-31.

29. Srinivasan S, Liu C, Mitchell CM, Fiedler TL, Thomas $\mathrm{KK}$, Agnew KJ, et al. Temporal variability of human vaginal bacteria and relationship with bacterial vaginosis. PloS one 2010;5(4):e10197.

30. Bailey RR. Significance of coagulase-negative Sta- phylococcus in urine. J Infect Dis 1973;127(2):179-82.

31. Villegas J, Schulz M, Soto L, Sanchez R. Bacteria induce expression of apoptosis in human spermatozoa. Apoptosis 2005;10(1):105-10.

32. Vieira OV, Hartmann DO, Cardoso CM, Oberdoerfer D, Baptista M, Santos MA, et al. Surfactants as microbicides and contraceptive agents: a systematic in vitro study. PloSone. 2008;3(8):e2913.

33. Lu Y, Bhushan S, Tchatalbachev S, Marconi M, Bergmann $\mathrm{M}$, Weidner $\mathrm{W}$, et al. Necrosis is the dominant cell death pathway in uropathogenic Escherichia coli elicited epididymo-orchitis and is responsible for damage of rat testis. PloSone 2013;8(1):e52919.

34. Vicari $E$, Mongioì $A$, Speciale $A$, Caccamo $F$, Calogero A, Gulizia S, et al. Enhancing detection of gonococcus in ejaculates of adult males using sperm dilution. Sys Biol Reprod Med 1986;16(1):19-23.

35. Comhaire F, Verschraegen G, Vermeulen L. Diagnosis of accessory gland infection and its possible role in male infertility. Int J Androl 1980;3(1):32-45.

36. Motrich RD, Sanchez L, Maccioni M, Mackern-Oberti JP, Rivero VE. Male rat genital tract infection with Chlamydia muridarum has no significant consequence on male fertility. JUrol 2012;187(5):1911-7.

37. Lozano-Hernandez R, Vivas-Acevedo G, Munoz de Vera MG. Mycoplasmas y anticuerpos anti-Chlamydia en semen de hombres infértiles y su relación con la calidad seminal y los marcadores de glándulas sexuales accesorias masculinas. Investigación Clínica 2012;53(2):138-47.

38. Levy R, Grattard F, Maubon I, Ros A, Pozzetto B. Bacterial risk and sperm cryopreservation. Andrologia 2004;36(5):282-5.

39. Zea-Mazo JW, Negrette-Mejia YA, Cardona-Maya W. Virus de transmisión sexual: relación semen y virus. Actas Urol Esp 2010;34(10):845-53.

40. Fowler JE, Jr., Mariano M. Bacterial infection and male infertility: absence of immunoglobulin A with specificity for common Escherichia coli 0-serotypes in seminal fluid of infertile men. J Urol 1983;130(1):171-4. 